\title{
Heterochromatin protein 1 forms distinct complexes to direct histone deacetylation and DNA methylation
}

\author{
Shinji Honda ${ }^{1,4}$, Zachary A Lewis ${ }^{1,4}$, Kenji Shimada ${ }^{2}$, Wolfgang Fischle ${ }^{3}$, Ragna Sack ${ }^{2}$ \& Eric U Selker ${ }^{1}$ \\ DNA methylation, methylation of histone $\mathrm{H} 3$ at Lys9 (H3K9me3) and hypoacetylated histones are common molecular features \\ of heterochromatin. Important details of their functions and inter-relationships remain unclear, however. In Neurospora crassa, \\ H3K9me3 directs DNA methylation through a complex containing heterochromatin protein 1 (HP1) and the DNA methyltransferase \\ DIM-2. We identified a distinct HP1 complex, HP1, CDP-2, HDA-1 and CHAP (HCHC), and found that it is responsible for silencing \\ independently of DNA methylation. HCHC defects cause hyperacetylation of centromeric histones, greater accessibility of DIM-2 \\ and hypermethylation of centromeric DNA. Loss of $\mathrm{HCHC}$ also causes mislocalization of the DIM-5 H3K9 methyltransferase at a \\ subset of interstitial methylated regions, leading to selective DNA hypomethylation. We demonstrate that HP1 forms distinct DNA \\ methylation and histone deacetylation complexes that work in parallel to assemble silent chromatin in N. crassa.
}

\begin{abstract}
Heterochromatin, the densely staining fraction of eukaryotic genomes, is refractory to DNA-based transactions such as transcription and recombination, but is functionally important. The relative 'darkness' of heterochromatin seems to be critical for normal centromere function ${ }^{1}$. Hallmarks of heterochromatin in higher organisms include a paucity of genes, an abundance of repetitive DNA, hypoacetylated histones $\mathrm{H} 3$ and $\mathrm{H} 4, \mathrm{H} 3 \mathrm{~K} 9 \mathrm{me}$, association of specific proteins such as HP1 and the presence of DNA methylation ${ }^{1,2}$. The filamentous fungus $N$. crassa also shows these features of heterochromatin and is convenient for genetic and biochemical studies. Research with N. crassa on the function and control of heterochromatin shows a direct connection between chromatin structure and DNA methylation ${ }^{3-5}$. The DIM-5 lysine methyltransferase trimethylates H3K9 (ref. 6) and this mark is then recognized by the chromodomain protein HP1, which recruits the DNA methyltransferase DIM-2 (ref. 7). Consistent with this pathway, DNA methylation, H3K9me3 and HP1 colocalize at centromeres, telomeres and dispersed transposon relics, all of which show evidence of the genome defense system repeat-induced point mutation (RIP) ${ }^{8}$.

In the yeast Schizosaccharomyces pombe, four of the crucial factors involved in heterochromatin formation contain chromodomains: the lysine methyltransferase Clr4, Chp1 (a component of RNA-induced transcriptional silencing complex) and the HP1 homologs Swi6 and Chp2 (refs. 1,9,10). Mammals and insects also contain multiple isoforms of HP1 that make distinct contributions in regulating heterochromatin ${ }^{1-14}$. Although N. crassa contains only one HP1 homolog 4 , it has several other chromodomain proteins. We therefore investigated whether candidate chromodomain proteins (designated CDP-1 to CDP-4 $)^{15}$ (Supplementary Fig. 1a) are involved in heterochromatin formation and DNA methylation.
\end{abstract}

\section{RESULTS}

Mutants lacking CDP-2 show abnormal DNA methylation

To explore the possibility that chromodomain proteins have a role in the control and function of DNA methylation and heterochromatin, we generated and tested chromodomain protein $(c d p)$ mutants for effects on DNA methylation at representative methylated genomic regions. A mutant lacking CDP-2 (Fig. 1a) affected DNA methylation in a manner not previously observed. In particular, in restriction digests with the 5-methylcytosine (5mC)-sensitive enzyme BfuCI, chromodomain protein-2 (cdp-2) strains showed hypomethylation at some loci and hypermethylation at others (Fig. 1b). Even comparisons of stained restriction digests of total genomic DNA showed more high-molecularmass DNA in $c d p-2$ strains (BfuCI digests in Fig. 1b; results for other $5 \mathrm{mC}$-sensitive enzymes in Supplementary Fig. 1b), suggesting that loss of CDP-2 causes global hypermethylation. By contrast, Southern hybridizations probed with several methylated regions (8:A6, 8:G3, 8:F10, 8:F3, $\Psi 63,9: \mathrm{E} 1$ and 5:B8 $)^{16}$ showed that the $c d p-2$ mutation caused a complete loss of DNA methylation at 8:A6 and a partial loss of DNA methylation at 8:G3, $\Psi 63$ and 9:E1. We found normal DNA methylation at 8:F10 and 5:B8 and a mixture of hyper- and hypomethylation at 8:F3 (for example, an extra high-molecular-mass band for the 8:F3 region in $c d p-2$ mutants indicates hypermethylated products; Fig. 1b and Supplementary Fig. 1c).

To test the generality of these observations, we examined the distribution of DNA methylation across an entire N. crassa chromosome by probing a high-density microarray for linkage group VII (LG VII) with immunoprecipitated methylated DNA (MeDIP) from wild-type and $c d p-2$ strains (Fig. 1c and Supplementary Fig. 2a,b $)^{8}$. We observed multiple examples of region-specific

\footnotetext{
${ }^{1}$ Department of Biology and Institute of Molecular Biology, University of Oregon, Eugene, Oregon, USA. ${ }^{2}$ Friedrich Miescher Institute for Biomedical Research, Basel, Switzerland. ${ }^{3}$ Laboratory of Chromatin Biochemistry, Max Planck Institute for Biophysical Chemistry, Göttingen, Germany. ${ }^{4}$ Present addresses: Life Science Unit, University of Fukui, Fukui, Japan (S.H.); Department of Microbiology, University of Georgia, Athens, Georgia, USA (Z.A.L.). Correspondence should be addressed to E.U.S. (selker@uoregon.edu).
} 
Figure 1 Aberrant DNA methylation in the $c d p-2$ mutant. (a) Schematic of CDP-2. (b) Genomic DNA from wild type (WT), dim-2 and $c d p-2$ was digested with $5 \mathrm{mC}$-sensitive $\mathrm{BfuCl}(\mathrm{B})$ or its $5 \mathrm{mC}$-insensitive isoschizomer Dpnll (D), gelfractionated, visualized by ethidium bromide $(\mathrm{EtBr})$ straining and analyzed by Southern hybridizations with indicated probes corresponding to representative methylated regions (8:A6, 8:G3, $8: F 10$ and 8:F3) ${ }^{16}$. An extra high-molecularmass band for the $8: \mathrm{F} 3$ region in $c d p-2$ mutants indicates hypermethylated products. (c) DNA methylation profile of wild-type and $c d p-2$ strains across $N$. crassa LG VII. The immunoprecipitation per input ratio for each oligo was plotted as a $\log _{2}$ value ( $y$ axis). In plots of microarray data, blue is RIP product index (ratio of number of TpA and ApT dinucleotides) and red is RIP substrate index (ratio of sum of $\mathrm{CpA}$ and $\mathrm{TpG}$ dinucleotides and sum of ApC and GpT dinucleotides) per (ApC + GpT) ratio). Regions with RIP show product index $>1.0$ and substrate index $<1.0$ (refs. 16,31). Black arrows, seven representative methylated regions of LG VII. Predicted genes, gray arrows; regions with moderate RIP, red bars. (d) DNA methylation profile at CenVII in wild-type (light blue) and $c d p-2$ (orange) strains. (e) Southern hybridizations confirming hypermethylation at LG VII centromere in $c d p-2$ (probes in $\mathbf{d}$ ).

hyper- and hypomethylation in the $c d p-2$ mutant, confirming and extending the Southern hybridization data. Almost half of the LG VII methylated regions (20 of 45) showed lower or no methylation in the $c d p-2$ strain. Most hypomethylated regions (15 of 20) were short and seemed to have undergone moderate RIP (based on calculated RIP indices; regions with RIP show product index $>1.0$ and substrate index $<1.0)^{16,31}$, whereas longer $(>10$ kilobase $(\mathrm{kb})$ ) regions showing more extensive RIP, such as the centromere

a

b

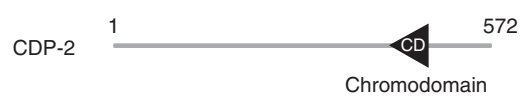

$$
\text { d }
$$$$
\begin{aligned}
& \text { d WT vs } \Delta c d p-2 \quad 20 \mathrm{~kb} \\
& \text { CenVIIL Cen'VIIM Cen'VIIR }
\end{aligned}
$$
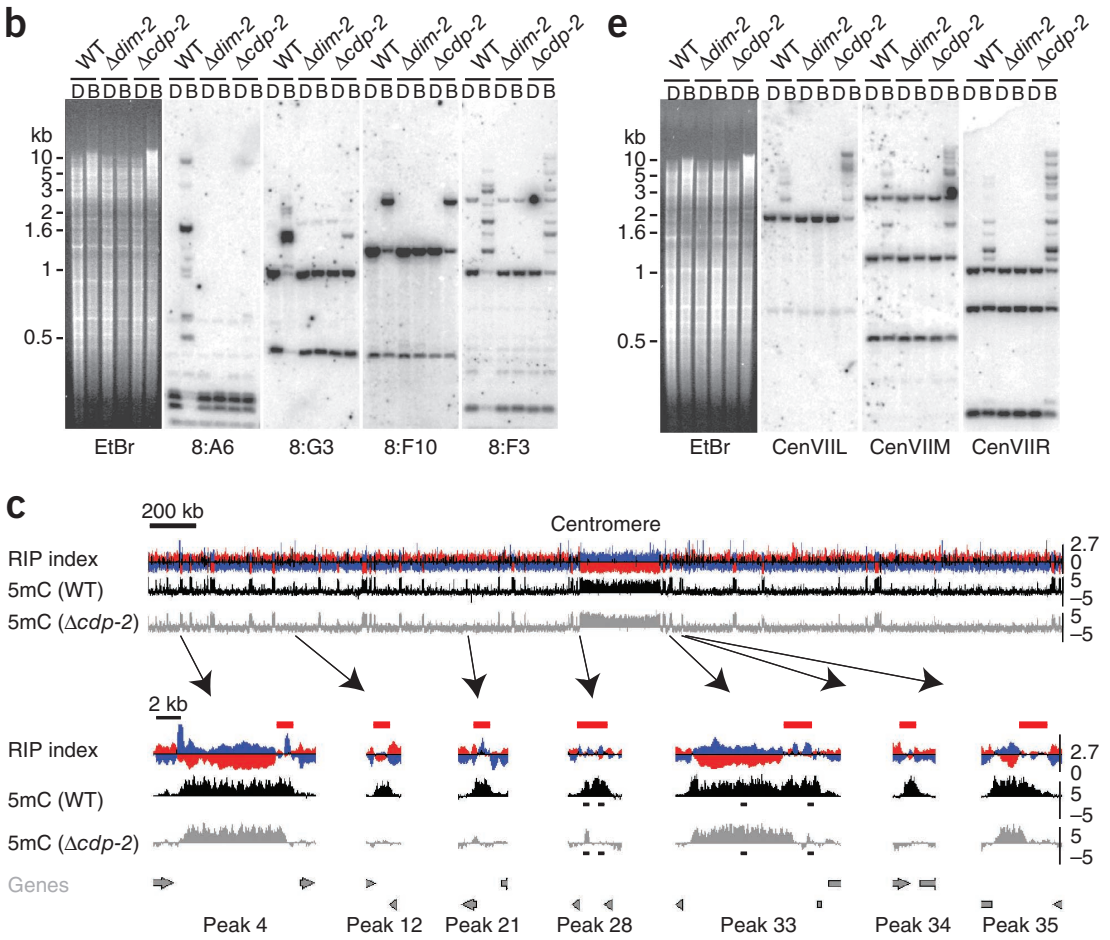
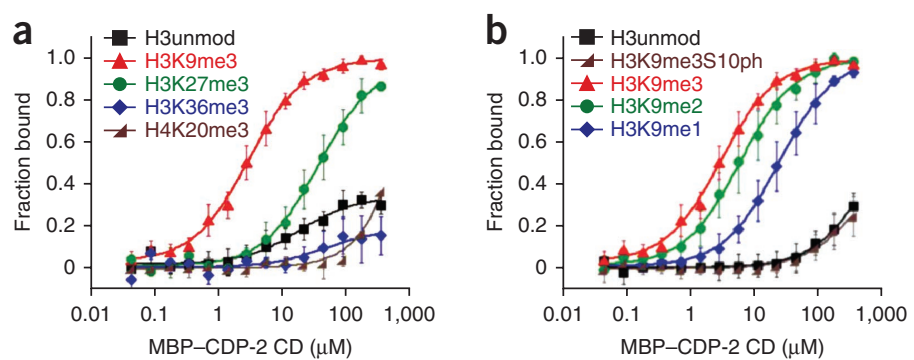

Figure 2 The CDP-2 chromodomain preferentially binds to H3K9 methylation and CDP-2 stability depends on HP1. (a, b) Fluorescence polarization binding assays of recombinant MBP-CDP-2 chromodomain (CD) with indicated fluoresceinated histone peptides. Average results from at least three independent measurements are plotted (mean \pm s.d.). (c) CDP-2 colocalizes with HP1. Conidia of indicated strains, which carried wild-type genes for CDP-2 and HP1 and genes for indicated chimeric proteins, were examined by microscopic analyses. Differential interference contrast (DIC), fluorescence (HP1-GFP and CDP-2-RFP) and overlay images. (d) CDP-2 localization depends on DIM-5 but not DIM-2. CDP-2-GFP localization was examined in $d_{i m}^{+}(\mathrm{WT}), \operatorname{dim}-5$ or dim-2 strains in addition to $c d p-2$ mutants carrying a functional CDP-2-GFP construct driven by the ccg-1 promoter at the his-3 locus. Functionality of the CDP-2-GFP construct was demonstrated in the $\mathrm{dim}^{+}$background by occurrence of normal DNA methylation (data not shown). (e) CDP-2 localization depends on HP1 but not vice versa. (f) CDP-2 stability depends on HP1. Extracts from strains expressing CDP-2-GFP in WT and hpo strains were analyzed by western blotting with antibodies to GFP.
C

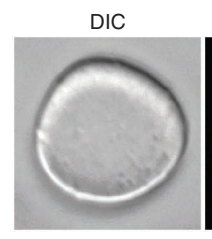

d

5
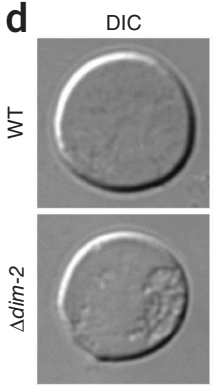

हो

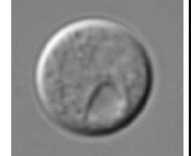

HP1-GFP

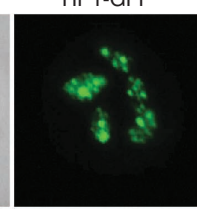

CDP-2-GFP
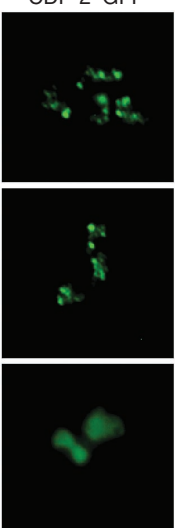

CDP-2-RFP

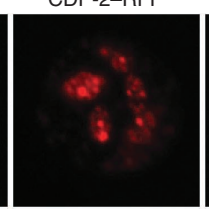

e

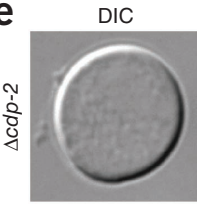

DIC

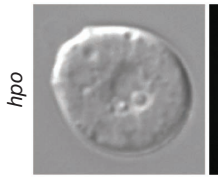

f

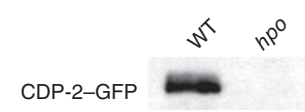

Overlay

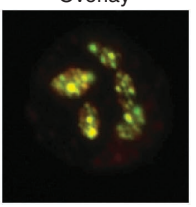

HP1-GFP

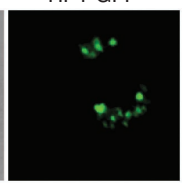

CDP-2-GFP

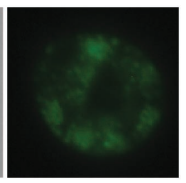

$r p^{0}$ region (Fig. 1d), retained or had greater methylation in the $c d p-2$ strain. We confirmed representative MeDIP results by Southern hybridizations (Supplementary Fig. 2c). After verifying hypermethylation at three centromere VII (CenVII) sequences (Fig. 1e), we tested all of the six other centromeres of $N$. crassa and found that they too were hypermethylated (Supplementary Fig. 2d), 
Figure 3 Mutation of $c d p-2$ causes lower H3K9me3 and HP1 localization at hypomethylated regions but no difference at hypermethylated regions compared with wild type. (a) Relative enrichment of $\mathrm{H} 3 \mathrm{~K} 9 \mathrm{me} 3$ at indicated regions for wild-type, hpo and $c d p-2$ strains. Peaks 28a, 28b, 33a and 33b are in Figure 1c or Figure 3c. A euchromatic gene lacking DNA methylation ( $h \mathrm{H} 4-1)$ was used as internal control. Ratios of intensities measured for $h$ H4- 1 and indicated probe were normalized to ratios obtained without immunoprecipitation (total input). (b) Relative enrichment of HP1-GFP at indicated regions for wild-type and cdp-2 strains. (c) Distribution of DNA methylation, H3K9 methylation and HP1 at peaks 28 and 33 for indicated strains. Data in a,b are mean and s.d. of independent biological replicates. suggesting that the global hypermethylation observed in $c d p-2$ mutants by ethidium bromide staining is predominantly due to centromere hypermethylation.

\section{CDP-2 chromodomain binds to $\mathrm{H} 3 \mathrm{~K} 9 \mathrm{me} 3$}

To test whether the CDP-2 chromodomain binds methylated histones, we carried out fluorescence polarization binding assays with a purified recombinant CDP-2 chromodomain and $\mathrm{H} 3$ or $\mathrm{H} 4$ peptides methylated on various lysines ${ }^{10,17}$. The CDP- 2 chromodomain bound strongly to $\mathrm{H} 3 \mathrm{~K} 9 \mathrm{me} 3$ and moderately to $\mathrm{H} 3 \mathrm{~K} 27 \mathrm{me} 3$, whereas it did not bind to H3K4me3, H3K36me3 or unmodified H3 (Fig. 2a). The CDP-2 chromodomain bound all methylated forms of H3K9, showing increasing affinity for mono-, di- and trimethylated H3K9 (Fig. 2b). Notably, this binding was prevented by phosphorylation of the adjacent serine (H3S10ph; Fig. 2b), as has been observed for the HP1 chromodomain ${ }^{18}$.

To investigate binding of the CDP-2 chromodomain to $\mathrm{H} 3 \mathrm{~K} 9 \mathrm{me} 3$ in vivo, we generated strains expressing C-terminal red fluorescent protein (RFP)- or green fluorescent protein (GFP)-tagged CDP-2 (CDP-2-RFP; CDP-2-GFP) in various strain backgrounds. Insertion of CDP-2-GFP into the his-3 locus of a $c d p-2$ mutant strain restored DNA methylation (data not shown), indicating that it was functional. The epitope-tagged CDP-2 localized to heterochromatin, as shown for example by its colocalization with foci of GFP-tagged HP1 (HP1-GFP), which binds to $\mathrm{H} 3 \mathrm{~K} 9 \mathrm{me} 3$ in vivo ${ }^{4}$ (Fig. 2c). We also found that the heterochromatic foci of CDP-2-GFP depends on the H3K9 methyltransferase DIM-5 (Fig. 2d), suggesting that CDP-2 localization depends, directly or indirectly, on $\mathrm{H} 3 \mathrm{~K} 9 \mathrm{me} 3$. By contrast, punctate localization of CDP-2-GFP was intact in the dim-2 strain (Fig. 2d), indicating that it does not depend on DNA methylation.

Considering that both HP1 and CDP-2 bind to, and depend on, $\mathrm{H} 3 \mathrm{~K} 9 \mathrm{me} 3$, we were interested in determining whether localization of either protein depends on the other. We therefore generated $c d p-2$

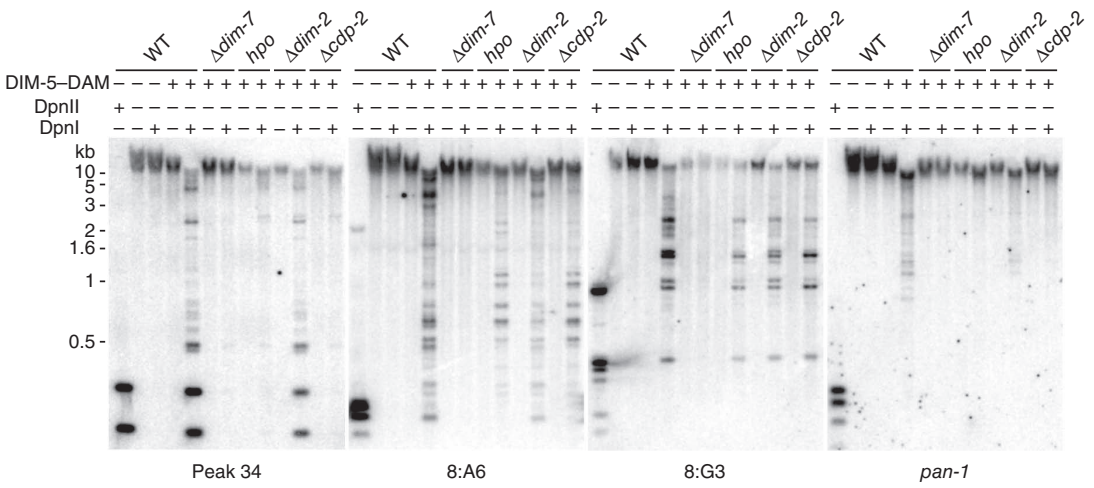

mutants expressing HP1-GFP and hpo (the gene encoding HP1) mutants expressing CDP-2-GFP and examined the localization of the GFP-tagged proteins microscopically. We observed normal foci of HP1-GFP in the $c d p-2$ mutant, indicating that HP1 does not depend on CDP-2 for its localization. By contrast, hpo strains expressing CDP-2-GFP showed weak and diffuse fluorescence, suggesting that CDP-2-GFP is unstable and/or mislocalized in the hpo mutant (Fig. 2e). To distinguish between these possibilities, we made cell extracts from wild-type and hpo strains expressing CDP-2-GFP and carried out western blot analyses using antibodies for GFP. CDP-2GFP was extremely unstable in $h p o$ mutants (Fig. 2f), whereas HP1 stability was not affected in $c d p-2$ strains (Supplementary Fig. 3a). We obtained similar results using a CDP-2-3 $\times$ Flag fusion protein (CDP-2-Flag) driven by the native $c d p-2$ promoter (Supplementary Fig. 3b), confirming that CDP-2 stability depends on HP1.

\section{CDP-2 is required for normal DIM-5 localization}

Because H3K9me3 directs DNA methylation in N. crassa and these two marks normally colocalize ${ }^{5,8}$, we investigated whether the hypo- and hypermethylation of DNA observed in $c d p-2$ strains reflects similar changes in $\mathrm{H} 3 \mathrm{~K} 9 \mathrm{me} 3$. Western blotting experiments did not show an effect of $c d p-2$ on H3K9me3 globally (Supplementary Fig. 3c) but chromatin immunoprecipitation (ChIP) experiments were informative. Regions of moderate RIP that showed a nearly complete loss of DNA methylation (for example, the 8:A6 region and the LG VII methylated peak $33 \mathrm{~b}$ and peak $28 \mathrm{~b}$ regions) showed markedly lower H3K9me3 and HP1 localization and regions that showed somewhat lower DNA methylation (for example, 8:G3 and peak 28a) showed moderately lower H3K9me3 and HP1 localization compared with wild type (Fig. 3a,b). Notably, knockout of the gene encoding HP1 ( $h p o$ ) showed nearly identical results (Fig. 3a). The regions most susceptible to loss of $\mathrm{H} 3 \mathrm{~K} 9 \mathrm{me} 3$ and DNA methylation seem to be those that normally show the most binding of HP1 (Fig. 3c and Supplementary

Fig. 4) ${ }^{8}$. This suggested that these two chromodomain proteins cooperate to maintain $\mathrm{H} 3 \mathrm{~K} 9 \mathrm{me} 3$ at some loci. In contrast to the changes in $\mathrm{H} 3 \mathrm{~K} 9 \mathrm{me} 3$ observed at hypomethylated regions of $c d p-2$ strains, there were no

Figure 4 CDP-2 is required for DIM-5 recruitment to regions with moderate RIP. DIM- 5 accessibility at indicated regions was assayed by DamID. Genomic DNA isolated from indicated strains was incubated with $(+)$ or without $(-)$ restriction enzyme. Southern blots were probed with hypomethylated peak 34 and 8:A6 regions, the slightly affected 8:G3 region and the euchromatic gene pan-1. 
Figure 5 Identification of HCHC. (a) Purification and mass spectrometric analyses of proteins associated with CDP-2. Extract from strain expressing CDP-2-HAT-Flag was treated by two-step purification ${ }^{21}$, separated by SDS-PAGE, visualized by Coomassie blue staining and analyzed by mass spectrometry. Positions of CDP-2-associated proteins are shown. Unlabeled bands were found to be background or degraded proteins. (b) HDA-1 associates with CDP-2 in vivo. WB, western blot. (c) CHAP associates with CDP-2 in vivo. (d) CDP-2 associates with HP1 but not DIM-2 in vivo. Coimmunoprecipitation (IP) analyses in $\mathbf{b}$ - $\mathbf{d}$ were done with antibodies to epitopes on extracts from strains expressing indicated tagged proteins.

changes in $\mathrm{H} 3 \mathrm{~K} 9 \mathrm{me} 3$ and HP1 localization at regions with high RIP that showed hypermethylation in $c d p-2$ mutants (Fig. 3a,b), suggesting that a step downstream of HP1 binding to H3K9me3 is involved in hypermethylation.

To determine whether recruitment of DIM- 5 to chromatin is altered in $c d p-2$ and hpo strains, we used the DamID method, in which an adenine methylase (Dam) marks DNA in the vicinity of a Dam-tagged protein ${ }^{19}$. We introduced a DIM-5-Dam fusion construct into the hpo, dim-2 and $c d p-2$ strains, the positive-control wild-type strain and the negative-control dim-7 strain (required for localization of DIM-5 $)^{20}$. Genomic DNA was digested with DpnI, which specifically cuts adenine-methylated GATC sites. Wild-type and dim-2 strains, but not dim-7 strains, showed low-molecular-mass fragments and some intermediate-molecular-mass fragments, indicating DIM-5Dam localization, at regions that became hypomethylated in the $c d p-2$ strain (peak 34 and 8:A6) and in a weakly affected region (8:G3; Fig. 4). DpnI digestion was substantially lower in $h p o$ and $c d p-2$ mutants at the hypomethylated regions, and most markedly in peak 34 , which lacked detectable DNA methylation in $c d p$-2 strains (Fig. 4 and Supplementary Fig. 2c). Region 8:A6, which did not lose all DNA methylation, showed a smaller effect, and almost no change was found at the weakly affected region (8:G3). As we expected, a probe for the unmethylated region (pan-1) hybridized almost exclusively to highmolecular-mass DNA corresponding to mainly undigested DNA in all strains (Fig. 4). These data suggest that CDP-2 and HP1 are required for DIM-5 targeting regions with moderate RIP.

\section{Distinct HP1 complexes}

To gain further insight into how CDP-2 controls DNA methylation, we purified CDP-2-interacting proteins by two-step affinity purification $^{21}$. The purified samples were gel-fractionated, visualized
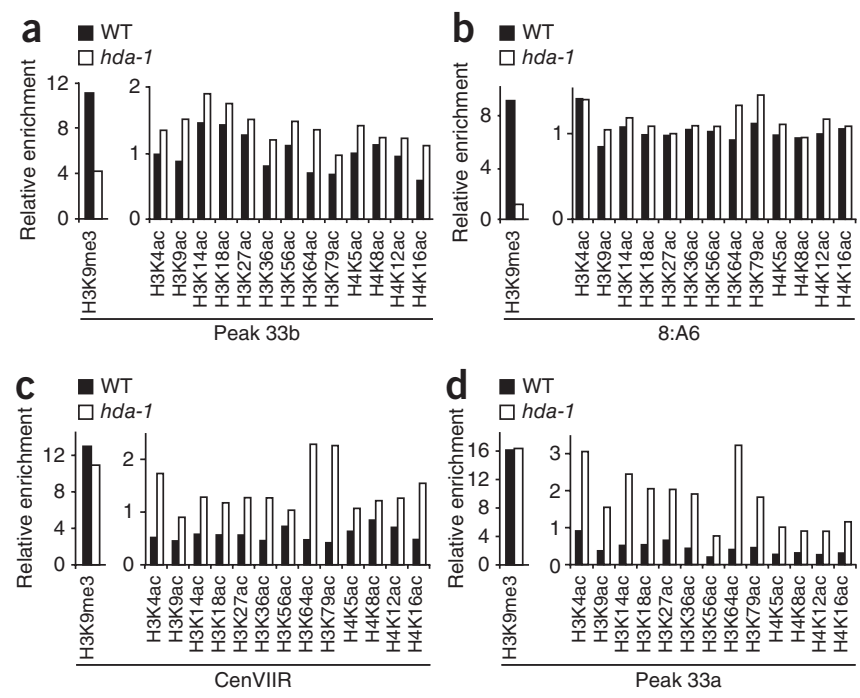

a

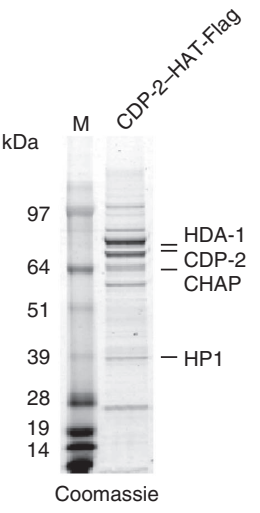

b
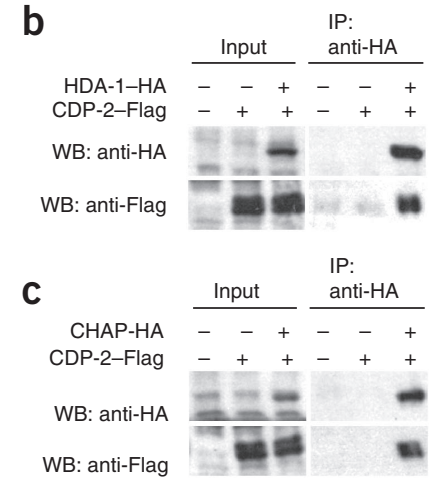

d

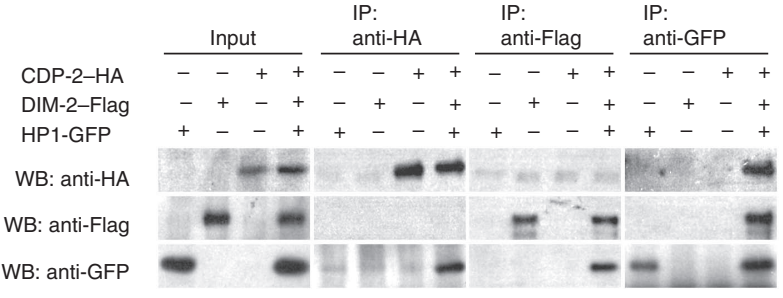

by Coomassie blue staining (Fig. 5a) and then analyzed by mass spectrometry. We identified CDP-2, HP1, the histone deacetylase HDA-1 (ref. 22) and a hypothetical protein encoded by NCU01796 (Supplementary Fig. 5a). The NCU01796 gene, which we have named CDP-2- and HDA-1 associating protein (chap), encodes a 552-residue protein containing two AT-hook motifs and two zinc finger motifs. To verify that $\mathrm{HDA}-1$ and $\mathrm{CHAP}$ interact with CDP-2, we created strains expressing CDP-2-Flag and HDA-1-hemagglutinin (HDA-1-HA) or CHAP-HA driven by their endogenous promoters and verified their interactions by coimmunoprecipitation (Fig. 5b,c). We also verified the interaction of HP1 with HDA-1 and CHAP (Supplementary Fig. 6a,b). To test whether HDA-1 and CHAP are also important for controlling DNA methylation, we carried out MeDIP and array-based hybridization (MeDIP-chip) and Southern hybridization analyses on hda-1 and chap knockout mutants (Supplementary Fig. 7a-c). The hda-1 mutant showed selective hypo- and hypermethylation equivalent to that of the $c d p-2$ strain. DNA methylation defects in the chap mutant were similar although somewhat less pronounced. DamID experiments showed mislocalization of DIM-5-Dam in a hypomethylated region in all three mutants, and in the HP1 mutant (Supplementary Fig. 7d). These data indicate that HP1, CDP-2, HDA- 1 and CHAP form a complex that controls DNA methylation and $\mathrm{H} 3 \mathrm{~K} 9$ methylation.

Our observation that purified CDP-2 associated with HP1, but not with the DIM-2 DNA methyltransferase, which forms a complex with HP1 (ref. 7), raised the question of whether HP1 forms mutually exclusive complexes with CDP-2 and DIM-2. To explore this possibility, we purified DIM-2 and identified interacting proteins by mass spectrometry. We detected HP1 but not CDP-2, HDA-1 or CHAP (Supplementary Fig. 5b). We next did a coimmunoprecipitation experiment to test directly whether HP1 forms mutually exclusive complexes with DIM-2 and CDP-2. We built a N. crassa strain expressing CDP-2-HA, DIM-2-Flag and HP1-GFP driven by

Figure $6 \mathrm{HCHC}$ is required for normal histone $\mathrm{H} 3$ and $\mathrm{H} 4$ acetylation. (a-d) Relative enrichment of $\mathrm{H} 3 \mathrm{~K} 9 \mathrm{me} 3$ and acetylated $\mathrm{H} 3$ and $\mathrm{H} 4$ determined by ChIP for wild-type and hda- 1 strains at hypomethylated regions peak $33 \mathrm{~b}$ (a) and $8: A 6$ (b) and at hypermethylated regions CenVIIR (c) and peak 33a (d). 


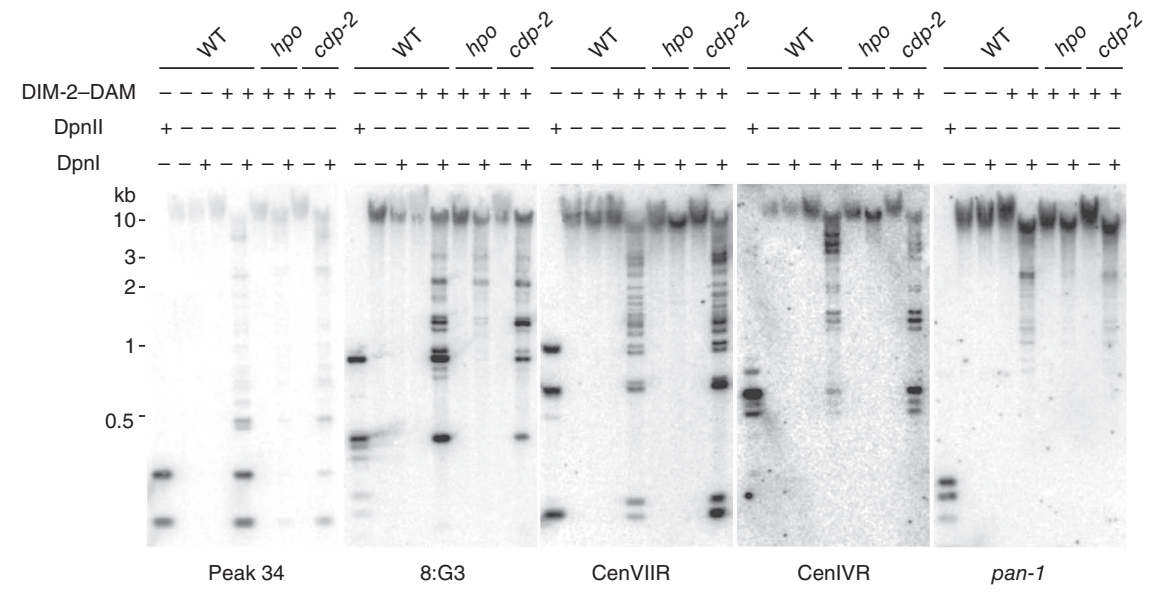

Figure 7 DIM-2 accessibility at centromere is enhanced in $c d p-2$ mutant. DIM-2 accessibility at indicated regions was assayed by DamID. Genomic DNA isolated from indicated strains was incubated with (+) or without (-) restriction enzyme. Southern blots were probed with slightly hypomethylated 8:G3 region, hypermethylated CenVIIR with high RIP and CenIVR and the euchromatic gene pan-1.

is altered in $c d p-2$ strains. To address this, we placed a DIM-2-Dam fusion construct at the native dim-2 locus of wild-type, $h p o$ and $c d p-2$ strains and compared the resulting adenine methylation in various genomic regions. The wild-type strains showed lowmolecular-mass fragments corresponding to

their endogenous promoters and used it to carry out pairwise coimmunoprecipitation tests. Both CDP-2-HA and DIM-2-Flag efficiently pulled down HP1-GFP. By contrast, CDP-2-HA did not pull down DIM-2-Flag; likewise, DIM-2-Flag did not pull down CDP-2 (Fig. 5d). Thus, these data verify that HP1 forms distinct complexes with CDP-2 and DIM-2. We refer to this newly discovered HP1 complex as $\mathrm{HCHC}$.

\section{HCHC shows regional effects on access of DIM-2 to chromatin} We considered several possible explanations for the regional hypermethylation observed in $c d p-2$ strains. One possibility was that in $c d p-2$ mutants, loss of CDP-2 would leave more HP1 available to bind DIM-2, leading to regional hypermethylation. However, we did not find enhanced interaction between DIM-2 and HP1 in $c d p-2$ mutants (Supplementary Fig. 6c). Moreover, the concentration of HP1 protein is about 20- to 30 -fold higher than those of DIM-2 and CDP-2 (Supplementary Fig. 6d), so CDP-2 probably does not appreciably compete with DIM-2 for HP1 binding. We next investigated whether the HCHC complex regulates DNA accessibility by directing histone deacetylation by HDA-1. We carried out ChIP experiments to examine $\mathrm{H} 3$ and $\mathrm{H} 4$ acetylation in the $h d a-1$ mutant and found that $\mathrm{H} 3$ and $\mathrm{H} 4$ acetylation was slightly greater at hypomethylated regions (for example, peak 33b and 8:A6; Fig. 6a,b). Notably, acetylation of $\mathrm{H} 3$ and $\mathrm{H} 4$ was even greater at hypermethylated regions (for example, CenVIIR and peak 33a; Fig. 6c,d) in the hda-1 strain. We observed similar enhanced acetylation in $h p o, c d p-2$ and chap mutants, but not dim-2 mutants, suggesting that all the components of $\mathrm{HCHC}$ are involved in histone deacetylation (Supplementary Fig. 8a-d).

Acetylation neutralizes the positive charge on the histones and decreases the interaction of histones with the negatively charged $\mathrm{DNA}^{23}$. This has been proposed to 'relax' chromatin structure, making it more accessible to nuclear proteins. We therefore investigated whether the accessibility of specific heterochromatin regions to DIM-2 fully digested DNA at all methylated regions, whereas DNA from the hpo strain was mainly undigested, consistent with our knowledge that DIM-2 localizes to these regions in an HP1-dependent manner (Fig. 7). Notably, we observed greater digestion at the hypermethylated regions (CenIVR and CenVIIR) in the $c d p-2$ strain, suggesting that DIM-2 occupancy was indeed greater at these regions (Fig. 7). As we expected, we did not observe DIM-2-Dam activity at the unmethylated region (pan-1). DIM-2-Dam activity was slightly lower at the moderately hypomethylated region (8:G3; Fig. 7) and was noticeably lower at the strongly hypomethylated region (peak 34; Fig. 7). We also observed greater DIM-2 accessibility at centromeric DNA in hda-1 and chap mutants compared with wild type (Supplementary Fig. 7e). These data suggest that $\mathrm{HCHC}$ regulates local access of DIM-2 to DNA.

\section{$\mathrm{HCHC}$ but not DIM-2 is responsible for centromere silencing}

Heterochromatic regions are generally assumed to be genetically silent. The indication that loss of HCHC leads to greater access to centromeric heterochromatin prompted us to test for centromeric silencing in N. crassa. Reasoning that HP1 may be required for silencing, we attempted to integrate the marker bar (Basta resistance) at centromere regions of an hpo strain by homologous recombination ${ }^{24}$. We successfully obtained Basta-resistant strains expressing the marker from several centromeric regions (CenIIIL::bar, CenVIR::bar and CenVIIM :: bar; data not shown). We then tested for silencing by adding back HP1, either by building heterokaryons with $h p o^{+}$strains or by crossing the marker into an $h p o^{+}$background. In both cases, silencing was established, providing evidence for centromeric silencing in N. crassa (Fig. 8a and Supplementary Fig. 8e). We also tested mutations in dim-2, $c d p-2, h d a-1$ and chap for possible effects on expression of centromeric reporters. Notably, centromeric silencing was lost in HCHC mutants but not in the dim-2 mutant, suggesting that $\mathrm{HCHC}$ is involved in centromeric silencing and that
Figure $8 \mathrm{HCHC}$ is required for centromere silencing independent of DNA methylation. (a) Serial dilutions of conidia from each of the indicated strains harboring a centromeric bar construct were spot-tested on medium with or without basta. (b) Linear growth rates for three wild-type (black), three dim-2 (red), three $c d p-2$ (blue) and three $c d p-2, d i m-2$ double mutant (brown). (c) Schematic of establishment and maintenance of heterochromatin in $N$. crassa. a

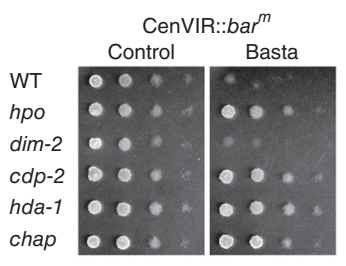

b

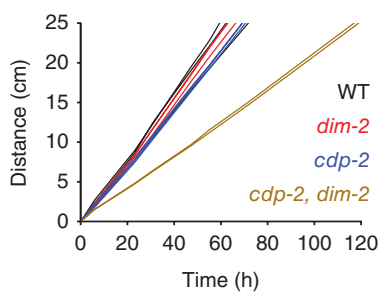

c

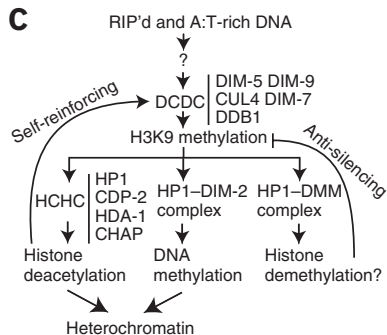


this silencing is independent of DNA methylation. Conversely, loss of DNA methylation per se, for example by silencing dim-2, is sufficient to reactivate methylated noncentromeric markers that have been tested ${ }^{20}$.

\section{Redundant functions of HCHC and HP1-DIM-2 complexes}

The coexistence of methylation-dependent and independent silencing in $N$. crassa suggested that these processes might serve partially redundant important functions. To test this, we isolated and analyzed the growth of the four classes of progeny from a cross of $c d p-2$ and dim-2 mutants: wild-type, dim-2, $c d p-2$ and $c d p-2 d i m-2$ double-mutant strains. The wild-type, dim-2 and $c d p-2$ strains had nearly identical growth rates (Fig. 8b). By contrast, the $c d p-2, d i m-2$ double mutants grew poorly (Fig. 8b). Similarly, the $h d a-1$, dim-2 and chap, dim-2 double mutants showed growth defects (Supplementary Fig. 8f,g). These data suggest that DNA methylation by the HP1DIM-2 complex and histone deacetylation by HCHC serve partially redundant functions in $N$. crassa.

\section{DISCUSSION}

We have identified a previously unknown silencing pathway in $N$. crassa that depends on a protein complex comprising the histone deacetylase HDA-1, a chromodomain protein, CDP-2, the abundant nuclear protein HP1 and a new protein, CHAP, which has two AT-hook motifs and two zinc finger motifs. Thus, $\mathrm{H} 3 \mathrm{~K} 9 \mathrm{me} 3$ is generated by the histone methyltransferase complex DIM-5, DIM-7, DIM-9, CUL4 and DDB1 complex (DCDC) ${ }^{25}$, which is recruited to AT-rich DNA, characteristic of RIP, by an unknown mechanism. This mark then directs DNA methylation and histone hypoacetylation through different HP1 complexes. The HP1-DIM-2 and HCHC complexes work in parallel to establish and maintain normal heterochromatin, and each serves as a partial backup system for the other (Fig. 8c). Meanwhile, HP1 mediates an opposing reaction through the putative histone demethylase complex DMM, which limits spreading of heterochromatin ${ }^{26}$. The balance of these three distinct HP1 complexes seems crucial for establishing and maintaining fully silenced heterochromatin at proper genomic locations (Fig. 8c).

We demonstrated the existence of centromeric silencing in $N$. crassa and showed that HCHC, but not DNA methylation, is required for this silencing. DNA in centromeric regions is hypermethylated. We did not detect greater $\mathrm{H} 3 \mathrm{~K} 9 \mathrm{me} 3$ in $c d p-2$, hda-1 or chap mutants but found marked hyperacetylation of histones $\mathrm{H} 3$ and $\mathrm{H} 4$ in these regions (Fig. 6). Perhaps the greater acetylation leads to a less condensed state of chromatin, which both relieves silencing and allows for greater accessibility of constitutive heterochromatin to DNA methyltransferase DIM-2 (Fig. 7). DNA methylation in the cores of centromeric regions is normally lower than that in smaller heterochromatin regions scattered elsewhere in the genome ${ }^{8,27}$. Notably, mutants lacking CDP-2, HDA-1 or CHAP show hypomethylation at several regions with moderate RIP in the noncentromeric chromosomal regions. This hypomethylation is caused by a failure to localize DIM- 5 to those regions, leading to loss of $\mathrm{H} 3 \mathrm{~K} 9 \mathrm{me} 3, \mathrm{HP} 1$ binding and DIM-2 recruitment. This is consistent with our earlier observation that a substantial, albeit small, fraction of $\mathrm{H} 3 \mathrm{~K} 9 \mathrm{me} 3$ and DNA methylation in the genome depends on HP1 localization ${ }^{8}$, suggesting feedback between HP1 and the methylation machinery. The mechanism of this feedback remains to be discovered. We did not detect interaction between HCHC and DIM-5, suggesting that $\mathrm{HCHC}$ is not required for direct recruitment of DIM-5 to those sites. Perhaps DCDC is sensitive to acetylation of an uncharacterized substrate of $\mathrm{HCHC}$, such as histone $\mathrm{H} 2 \mathrm{~B}$, which seems to be a major target for HDA-1 (ref. 22).
Although the fission yeast $S$. pombe lacks DNA methylation, it also uses multiple chromodomain proteins to maintain the structure, extent and function of heterochromatin. The N. crassa $\mathrm{HCHC}$ complex shares features with the $S$. pombe SHREC complex, which contains the HDAC Clr3 and at least one HP1 homolog (Chp2 and perhaps Swi6) ${ }^{11,13,14,28}$, but obvious differences exist. For example, CDP-2 and CHAP, which are conserved in filamentous fungi, are not evident in yeasts. In addition, a SNF2 chromatin-remodeling factor, Mit1, is an integral component of SHREC, whereas the homologous protein of $\mathrm{N}$. crassa is not found in the HCHC complex. There are also parallels between our observations and those in higher organisms. Most notably, in mammals the DNA methylation machinery shares the burden of silencing endogenous retrovirus-like elements with the DNA methylation-independent ESET-KAP1 silencing complex, which controls $\mathrm{H} 3 \mathrm{~K} 9$ methylation and contains $\mathrm{HP} 1$ and the NuRD histone deacetylase $\mathrm{e}^{29,30}$.

\section{METHODS}

Methods and any associated references are available in the online version of the paper at http://www.nature.com/nsmb/.

Note: Supplementary information is available on the Nature Structural \& Molecular Biology website.

\section{ACKNOWLEDGMENTS}

We thank L.L. David and members of his proteomics facility at Oregon Health Sciences University for identifying DIM-2-associated proteins and T. Khalafallah for technical support in preliminary work. E.U.S. thanks S. Gasser and D. Schübeler (F. Miescher Institute) and G. Almouzni (Institut Curie) for hosting him during a sabbatical. This work was funded by grant GM025690 to E.U.S. from the US National Institutes of Health. We acknowledge the Neurospora Genome Project and the Fungal Genetic Stock Center for providing materials.

\section{AUTHOR CONTRIBUTIONS}

S.H. and E.U.S. designed the research. Z.A.L. and E.U.S. carried out the MeDIP-chip experiments; E.U.S. and K.S. carried out CDP-2 purification; R.S. carried out mass spectrometry to identify CDP-2-associated proteins; W.F. carried out anisotropic binding assays to determine the CDP-2 chromodomain binding activity and S.H. carried out the other experiments. S.H., Z.A.L. and E.U.S. analyzed the data; S.H. and E.U.S. wrote the paper and Z.A.L., K.S. W.F. and R.S. edited the paper.

\section{COMPETING FINANCIAL INTERESTS}

The authors declare no competing financial interests.

Published online at http://www.nature.com/nsmb/.

Reprints and permissions information is available online at http://www.nature.com/ reprints/index.html.

1. Grewal, S.I. \& Jia, S. Heterochromatin revisited. Nat. Rev. Genet. 8, 35-46 (2007).

2. Beisel, C. \& Paro, R. Silencing chromatin: comparing modes and mechanisms. Nat. Rev. Genet. 12, 123-135 (2011).

3. Selker, E.U. Trichostatin A causes selective loss of DNA methylation in Neurospora. Proc. Natl. Acad. Sci. USA 95, 9430-9435 (1998).

4. Freitag, M., Hickey, P.C., Khlafallah, T.K., Read, N.D. \& Selker, E.U. HP1 is essential for DNA methylation in Neurospora. Mol. Cell 13, 427-434 (2004).

5. Tamaru, H. \& Selker, E.U. A histone H3 methyltransferase controls DNA methylation in Neurospora crassa. Nature 414, 277-283 (2001).

6. Tamaru, H. et al. Trimethylated lysine 9 of histone $\mathrm{H} 3$ is a mark for DNA methylation in Neurospora crassa. Nat. Genet. 34, 75-79 (2003).

7. Honda, S. \& Selker, E.U. Direct interaction between DNA methyltransferase DIM-2 and HP1 is required for DNA methylation in Neurospora crassa. Mol. Cell. Biol. 28, 6044-6055 (2008).

8. Lewis, Z.A. et al. Relics of repeat-induced point mutation direct heterochromatin formation in Neurospora crassa. Genome Res. 19, 427-437 (2009).

9. Schalch, T. et al. High-affinity binding of Chp1 chromodomain to K9 methylated histone $\mathrm{H} 3$ is required to establish centromeric heterochromatin. Mol. Cell 34 36-46 (2009).

10. Zhang, K., Mosch, K., Fischle, W. \& Grewal, S.I. Roles of the Clr4 methyltransferase complex in nucleation, spreading and maintenance of heterochromatin. Nat. Struct. Mol. Biol. 15, 381-388 (2008). 
11. Fischer, T. et al. Diverse roles of HP1 proteins in heterochromatin assembly and functions in fission yeast. Proc. Natl. Acad. Sci. USA 106, 8998-9003 (2009).

12. Hediger, F. \& Gasser, S.M. Heterochromatin protein 1: don't judge the book by its cover. Curr. Opin. Genet. Dev. 16, 143-150 (2006).

13. Motamedi, M.R. et al. HP1 proteins form distinct complexes and mediate heterochromatic gene silencing by nonoverlapping mechanisms. Mol. Cell 32, 778-790 (2008)

14. Sadaie, M. et al. Balance between distinct HP1 family proteins controls heterochromatin assembly in fission yeast. Mol. Cell. Biol. 28, 6973-6988 (2008).

15. Borkovich, K.A. et al. Lessons from the genome sequence of Neurospora crassa: tracing the path from genomic blueprint to multicellular organism. Microbiol. Mol. Biol. Rev. 68, 1-108 (2004).

16. Selker, E.U. et al. The methylated component of the Neurospora crassa genome. Nature 422, 893-897 (2003).

17. Jacobs, S.A. et al. Specificity of the HP1 chromo domain for the methylated $\mathrm{N}$-terminus of histone H3. EMBO J. 20, 5232-5241 (2001).

18. Fischle, W. et al. Regulation of HP1-chromatin binding by histone $\mathrm{H} 3$ methylation and phosphorylation. Nature 438, 1116-1122 (2005).

19. Vogel, M.J., Peric-Hupkes, D. \& van Steensel, B. Detection of in vivo protein-DNA interactions using DamID in mammalian cells. Nat. Protoc. 2, 1467-1478 (2007).

20. Lewis, Z.A., Adhvaryu, K.K., Honda, S., Shiver, A.L. \& Selker, E.U. Identification of DIM-7, a protein required to target the DIM-5 H3 methyltransferase to chromatin. Proc. Natl. Acad. Sci. USA 107, 8310-8315 (2010).

21. Honda, S. \& Selker, E.U. Tools for fungal proteomics: multifunctional Neurospora vectors for gene replacement, protein expression and protein purification. Genetics 182, 11-23 (2009).
22. Smith, K.M. et al. H2B- and H3-specific histone deacetylases are required for DNA methylation in Neurospora crassa. Genetics 186, 1207-1216 (2010).

23. Narlikar, G.J., Fan, H.Y. \& Kingston, R.E. Cooperation between complexes that regulate chromatin structure and transcription. Cell 108, 475-487 (2002).

24. Ninomiya, Y., Suzuki, K., Ishii, C. \& Inoue, H. Highly efficient gene replacements in Neurospora strains deficient for nonhomologous end-joining. Proc. Natl. Acad. Sci. USA 101, 12248-12253 (2004).

25. Lewis, Z.A. et al. DNA methylation and normal chromosome behavior in Neurospora depend on five components of a histone methyltransferase complex, DCDC. PLoS Genet. 6, e1001196 (2010)

26. Honda, S. et al. The DMM complex prevents spreading of DNA methylation from transposons to nearby genes in Neurospora crassa. Genes Dev. 24, 443-454 (2010).

27. Smith, K.M., Phatale, P.A., Sullivan, C.M., Pomraning, K.R. \& Freitag, M Heterochromatin is required for normal distribution of Neurospora crassa CenH3. Mol. Cell. Biol. 31, 2528-2542 (2011).

28. Sugiyama, T. et al. SHREC, an effector complex for heterochromatic transcriptional silencing. Cell 128, 491-504 (2007).

29. Sripathy, S.P., Stevens, J. \& Schultz, D.C. The KAP1 corepressor functions to coordinate the assembly of de novo HP1-demarcated microenvironments of heterochromatin required for KRAB zinc finger protein-mediated transcriptional repression. Mol. Cell. Biol. 26, 8623-8638 (2006).

30. Matsui, T. et al. Proviral silencing in embryonic stem cells requires the histone methyltransferase ESET. Nature 464, 927-931 (2010).

31. Margolin, B.S. et al. A methylated Neurospora 5S rRNA pseudogene contains a transposable element inactivated by repeat-induced point mutation. Genetics 149 , 1787-1797 (1998) 


\section{ONLINE METHODS}

Neurospora crassa strains and molecular analyses. All N. crassa strains and primers used in this study are listed in Supplementary Tables 1 and 2, respectively. The $c d p-2, h d a-1$ and chap knockout strains were constructed by the Neurospora Genome Project and were obtained from the Fungal Genetic Stock Center $^{32}$. Strains were grown, crossed and maintained according to standard procedures $^{33}$. DNA isolation, Southern blotting, histone isolation, western blotting, coimmunoprecipitation, fluorescence microscopy, $\mathrm{ChIP}^{7}$, two-step purification using an HAT-Flag tagged protein ${ }^{21}$ and MeDIP microarray assays ${ }^{8}$ were carried out as described. The construction of epitope-tagged proteins is described in Supplementary Methods. Immunoprecipitation, ChIP and western blotting were carried out with antibodies to Flag (Sigma, F3165), HA (mouse, University of Oregon monoclonal facility; rat, clone 3F10, Roche), H3K4me2 (Active Motif, 39142), H3K9me3 (Active Motif, 39161) H3K4ac (Active Motif, 39381), H3K9ac (Millipore, 07-353), H3K14ac (Diagenode, PAb-AHAHS-044), H3K18ac (Active Motif, 39587), H3K27ac (Active Motif, 39133), H3K36ac (Active Motif, 39379), H3K54ac (Active Motif, 39281), H3K64ac (Active Motif, 39545), H3K79ac (Active Motif, 39565), H4K5ac (Active Motif, 39583), H4K8ac (Diagenode, CS-103-100), H4K12ac (Active Motif, 39165) and H4K16ac (Active Motif, 39167). Ratios of signals for a gene lacking DNA methylation ( $h H 4-1)$ and the indicated probe were normalized to signals for total input and results of duplicate ChIP experiments were averaged.

Generation of recombinant CDP-2 chromodomain proteins and fluorescence polarization binding assays. The $c d p-2$ ORF (residues 397-572) was amplified with primers 3153 and 3154, digested with BamHI and SalI and inserted between sites for these enzymes in pMALc2 (New England Biolabs), yielding pTTK33. Purification of the MBP-CDP-2 chromodomain protein from Escherichia coli strain BL21 carrying PTTK33 was done as described by the manufacturer of pMALc2 and used in fluorescence-based anisotropy binding assays using the designated peptides ${ }^{10,18,34}$.

MeDIP-chip and ChIP-chip analyses. ChIP, MeDIP and microarray experiments were done as described ${ }^{8}$. DNA from immunoprecipitate and input fractions were differentially labeled and used to probe $\sim 40,000$ oligonucleotide sequences on an Agilent slide ${ }^{8}$.

Generation of a DIM-2-Dam knock-in construct at the dim-2 native locus. The E. coli dam gene was amplified from plasmid pCmyc-Dam ${ }^{19}$ with forward primer 3149, which contains a PacI site, and reverse primer 3150, which contains an XmnI site. The PCR products were digested with PacI and XmnI and

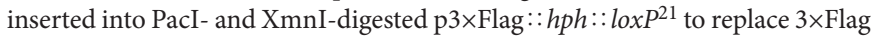
with Dam, yielding pDam ::hph::loxP. For DIM-2-DAM constructs, we modified our knock-in procedure ${ }^{21}$. Briefly, we first amplified two fragments by PCR with primers 1988, 1989, 1990 and 2013 as described for the DIM-2-Flag knock-in construct $^{7}$. We then mixed two gel-purified PCR products and $\mathrm{pDam}:: h p h::$ loxP and carried out PCR with primers 1988 and 1990 to assemble three products, which contained some overhanging DNA previously used in the yeast homologous recombination system. The assembled PCR products were gel-purified and introduced into N. crass $^{35}$. DamID was carried out as described ${ }^{19,20}$. Briefly, DNA samples were incubated with or without DpnI, which cuts adeninemethylated GATC sites. As a control for completely digested DNA, wild-type genomic DNA was incubated with the methylcytosine-insensitive isoschizomer DpnII. Digested DNA was analyzed by Southern hybridization with probes for representative regions that become hypomethylated or hypermethylated in the $c d p-2$ strain and the unmethylated euchromatic gene, pan-1.

DIM-2-Flag purification. $3 \times$ Flag-tagged proteins were purified essentially as described for yeast ${ }^{36}$. Conidia from a $250-\mathrm{ml}$ flask of a DIM-2-3×Flag strain (N3323) were grown overnight in $1,000 \mathrm{ml}$ of medium in a $2.8-1$ flask at $32^{\circ} \mathrm{C}$ with shaking. The harvested tissue ( $20 \mathrm{~g})$ was suspended in $50 \mathrm{ml}$ of ice-cold HC buffer (150 mM HEPES, pH 7.5, $250 \mathrm{mM} \mathrm{KCl,} 1 \mathrm{mM}$ EDTA, 10\% (v/v) glycerol, $0.02 \%(\mathrm{v} / \mathrm{v}) \mathrm{NP}-40$ ) with protein inhibitor cocktail (Roche), and extracts were generated by three cycles of sonication (Branson Sonifier-450; $1 \mathrm{~min}$ at 10 min intervals; duty cycle 80 ; output 3 ) and centrifuged at $10,000 \mathrm{~g}$ for $20 \mathrm{~min}$ at $4{ }^{\circ} \mathrm{C}$. The supernatant was collected and precleared with $250 \mu$ l Protein A agarose (Sigma) for $30 \mathrm{~min}$ and then incubated with $200 \mu \mathrm{l}$ anti-Flag M2 affinity resin (Sigma) for $3 \mathrm{~h}$. Purified samples were washed with HC buffer and eluted twice with $300 \mu \mathrm{l} \mathrm{HC}$ buffer containing $200 \mu \mathrm{g} \mathrm{ml}^{-1} 3 \times$ Flag peptide (Sigma). The samples were precipitated with trichloroacetic acid and analyzed by $\mathrm{MS}^{25,26}$.

Insertion of bar marker at centromeres. The bar gene, under the control of the trpC promoter, was amplified by PCR with pBARKS1 (ref. 37) as the template. We selected nonrepetitive regions of $N$. crassa centromeres to insert a marker gene by homologous recombination and used the computer program BLAST (http://www. broadinstitute.org/annotation/genome/neurospora/MultiHome.html) to confirm absence of similar sequences in the $N$. crassa genome sequence. We then amplified two adjacent $\sim 1-\mathrm{kb}$ regions of a nonrepetitive centromeric DNA from VIR by PCR with primers 3113, 3728, 3729 and 3730, which contain 29 nucleotides of DNA at each end of the bar gene. We then mixed three gel-purified PCR products, two adjacent cenVIR regions plus the bar gene, and carried out PCR with primers 3113 and 3730 to assemble the products (cenVIR::bar). The cenIIIL:: bar and cenVIIM :: bar constructs were similarly generated with primers $3725,3726,3727$ and 3100 and 2543, 3733, 3734 and 2544, respectively. The assembled constructs were transformed into a $\Delta m u s-52, h p o$ strain (N3018) by electroporation and a resulting transformant was characterized and then crossed with a mus-52 ${ }^{+}, h p o^{+}$ strain to recover progeny with the wild-type $m u s-52$ and hpo alleles.

32. Colot, H.V. et al. A high-throughput gene knockout procedure for Neurospora reveals functions for multiple transcription factors. Proc. Natl. Acad. Sci. USA 103, 10352-10357 (2006).

33. Davis, R.H. Neurospora: Contributions of a Model Organism (Oxford Univ. Press, 2000).

34. Jacobs, S.A., Fischle, W. \& Khorasanizadeh, S. Assays for the determination of structure and dynamics of the interaction of the chromodomain with histone peptides. Methods Enzymol. 376, 131-148 (2004).

35. Margolin, B.S., Freitag, M. \& Selker, E.U. Improved plasmids for gene targeting at the his-3 locus of Neurospora crassa by electroporation. Fungal Genet. Newsl. 44, 34-36 (1997).

36. Jia, S., Kobayashi, R. \& Grewal, S.I. Ubiquitin ligase component Cul4 associates with Clr4 histone methyltransferase to assemble heterochromatin. Nat. Cell Biol. 7, 1007-1013 (2005).

37. Pall, M.L. \& Brunelli, J.P. A series of six compact fungal transformation vectors containing polylinkers with multiple unique restriction sites. Fungal Genet. Newsl. 40, 59-62 (1993). 